\title{
An experimental study of the effect of surface morphology on squeal occurrence
}

\author{
Mayu Asano ${ }^{1}$, Jean-François Brunel ${ }^{2}$, Alexandre Mège-Revil ${ }^{1}$, Huawei Chen ${ }^{3}$, Xiandong \\ Liu $^{3}$, Mohamed Ichchou ${ }^{4}$ and Yannick Desplanques ${ }^{1}$
}

\author{
${ }^{1}$ Laboratoire de Mécanique de Lille - École Centrale de Lille - Cité Scientifique, 59651 Villeneuve d'Ascq, \\ France \\ 2 Laboratoire de Mécanique de Lille - École Polytechnique Universitaire de Lille (Polytech Lille), 59651 \\ Villeneuve d'Ascq, France \\ ${ }^{3}$ School of Mechanical Engineering, Beihang University, No. 37 Xueyuan Road, Haidian District, Beijing, China \\ ${ }^{4}$ Laboratoire de Tribologie et de Dynamique des Systèmes, Ecole Centrale de Lyon, 36 avenue Guy de \\ Collongue, 69134 Ecully Cedex, France
}

\begin{abstract}
Squealing is a major matter of concern for environmental issues and the raised expectations for superior performances in braking systems. Squealing, as well as other types of noise nuisances, contributes to noise pollution and leads to additional expenses for phonic isolation. Brake squeal noise, which is defined as noise at frequency higher than $1000 \mathrm{~Hz}$, occurs if the system has very high amplitude of mechanical vibration with sound pressure level above $80 \mathrm{~dB}$. It involves instable vibrations, which are quite well understood as a result of mode couplings between the rubbing parts. These mode couplings are allowed by modal frequency shifts induced by the changes of sliding contact conditions. Nonetheless, the origin of squeal occurrence remains misunderstood as many parameters have to be taken into account, making it virtually impossible to predict when a squeal noise will appear or disappear. The strong coupling between structure and material, the continual change of the materials surfaces (chemistry and morphology), the influence of external parameters (temperature, pressure, hygrometry) all play a role in squealing. As a consequence, research on squealing is empirical by nature. Following this, one of the main goals in braking sciences is to determine the set of conditions on the parameters that provoke the start or the end of a squealing noise.
\end{abstract}

In order to understand better this phenomenon, a simplified and original setup was devised. Pin-on-disc experiments were carried out in which the pin was a $1 \mathrm{~cm}^{2}$ square part of steel or aluminium mounted on a soft layer of siliconebased caulk to ensure the softness of the contact. Owing to the silicon thick layer, the normal load can be said to be equally distributed on the whole surface of the sample. Preliminary experiments showed that a normal load of $100 \mathrm{~N}$ was a good compromise to allow a good contact without observing plastic strain on the silicone.

The samples tested in this study are one polished aluminium part and three parts of the same steel (C45) textured differently by machining: rough polishing (RPS), ultrasonic vibration strengthening (UVS, sharkskin biomimetic texture) and ultrasonic vibration extrusion (UVE, thin parallel rays).

A glass disc was used because of its chemical inertia and transparency. Indeed, as its chemical reactivity is very low, there is virtually no risk of inducing chemical reactions that would provoke major changes in the nature and morphology of the surface. Moreover, a camera was used to film the evolution of the contact from above, the pin being underneath the disc. The contact is thus filmed through the glass disc.

Four two minute tests (acceleration phase, constant speed phase and deceleration phase) were performed on each sample. The results show that machining different morphologies on the surface on the same steel leads to very different results in terms of noise level and frequency. Indeed, for a given sample, the tangential load and the coefficient of friction stay quite constant during the test, but major variations are observed from one texture to another as the coefficient of friction varies from 0.20 for the RPS and 0.60 for the UVS. In the same way, the visible amount of third body was directly correlated with the squealing. Samples that produce more third body squeal more in terms of noise level and of number of frequencies involved. The frequencies are the same for all the steel samples, but there are a few more frequencies that appear in the UVS experiments.

As a consequence, the surface and distribution of the load bearing area seems to be crucial in the production of squeal. Moreover, experiments on aluminium have shown that the apparition of squealing would be linked on the one hand to the abrasion of the glass disc, and on the other hand to the instability of the third body layer that bears the load. In this case, the third body is composed of glass particles that aggregate in agglomerates of various size and shape. Five phases can be distinguished during these tests (fig. 1). In the first period corresponding to the acceleration of the disc, the disc is abraded on a few distinct radii, which leads to the constitution of a primary third body layer. The squealing noise registered during this first phase increases gradually with the speed and the amount of third body. The second phase starts when the abrasion is limited by the fact that the contact is born by the aggregated third body layer. No squealing was registered during this phase. Suddenly, as the third body layer that bears the load reaches a critical size, aggregates of third body get detached from the main mass and circulate in the contact until they reach the exit of 


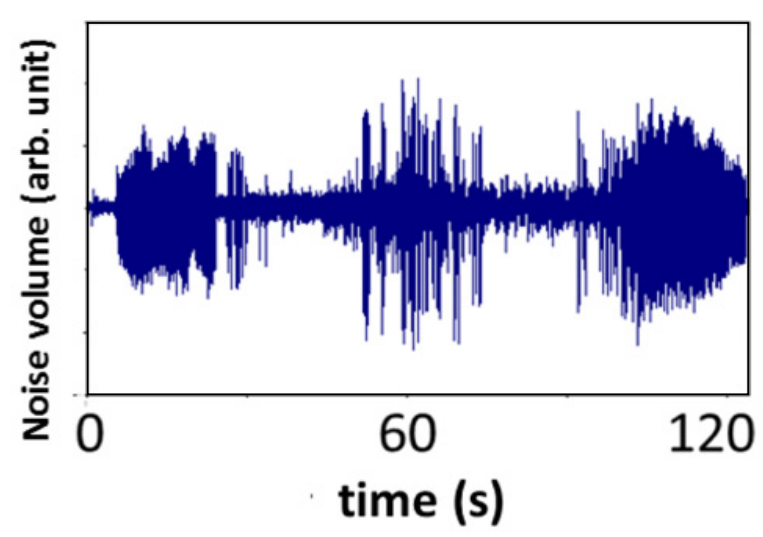

Figure 1. Microphone registering of the $\mathrm{Al}$ pin rubbing on glass disc.

the contact. This sporadically provokes the abrasion of the disc on new radii, and thus the emission of sharp and short squeal noises. In the fourth phase, both abrasion and squeal are limited. The third body layer bears the load anew, partly on the same area as previously, and partly on the newly abraded areas. Some of the third body also accumulates in the contact entrance without being able to get back in it. The fifth and last phase is linked with the deceleration of the disc until its complete stop. Once again abrasion of the disc and squeal are observed, but they occur quite continuously as in the first phase, though with decreasing intensity this time. The deceleration obviously generates instabilities in the third body layer, which would contribute to the production of squeal.

Squealing would be linked with the instabilities induced by abrasion phenomena, stable as well as metastable. Moreover, for steel samples the optical microscopy observations have shown areas where the metal has been strained thoroughly but not torn. As the third body is mainly made of glass, it would be interesting to determine the process implied in the aggregation of the micron-sized particles of glass into much bigger agglomerates of various densities. 